\title{
LA OXITOCINA EN EL VÍNCULO HUMANO-PERRO: REVISIÓN BIBLIOGRÁFICA Y ANÁLISIS DE FUTURAS ÁREAS DE INVESTIGACIÓN*
}

\author{
OXYTOCIN IN THE hUMAN-DOG BOND: REVIEW OF THE LITERATURE AND ANALYSIS OF \\ FUTURE INVESTIGATION FIELDS
}

\author{
Marcos Díaz Videla* y Pablo Adrián López ${ }^{* *}$
}

\begin{abstract}
${ }^{*}$ Trabajo realizado en el marco de la tesis doctoral del primer autor.
**Licenciado y Doctorando en Psicología. Docente de la Universidad de Flores (UFLO) e Integrante del Laboratorio de Investigación de la Escuela Sistémica Argentina. E-Mail: mdiazvidela@hotmail.com; antrozoologia@gmail.com

${ }^{* * *}$ Médico. Médico Neurólogo de los Servicios de Neurología y Neurofisiología del Hospital Alemán y Docente de la Universidad de Buenos Aires (UBA) y de la Universidad Católica Argentina (UCA).

Paraguay 4252, (1425) Ciudad Autónoma de Buenos Aires, Argentina.
\end{abstract}

\section{REsUmen}

El perro (Canis familiaris) ha sido la primera especie domesticada por el hombre como resultado de un proceso interactivo de miles de años, el cual produjo relaciones de competencia, cooperación y coevolución. Durante este proceso, los perros han adquirido habilidades comunicativas que favorecieron su relación con los humanos, la cual representa una expresión de apego. El rol de la oxitocina ha sido destacado en la formación de los vínculos de apego y en los comportamientos prosociales que facilitan las relaciones intraespecies. Se realizó una revisión sistemática de las investigaciones focalizadas en los efectos de la oxitocina sobre las interacciones entre humanos y perros. Se discuten los resultados en función de hallazgos de investigaciones referidas a la oxitocina y sus efectos intraespecies, así como en función de desarrollos en Antrozoología en general. Se destaca el posible rol de la oxitocina en distintos aspectos del vínculo humano-perro, como el efecto mascota, la cercanía emocional, el antropomorfismo y las respuestas a las características neoténicas. Finalmente, se describen posibles campos de investigación.
Palabras clave: Antrozoología; Apego; Oxitocina; Perro; Vínculo humano-animal.

\section{ABSTRACT}

Dog (Canis familiaris) has been the first species to be domesticated by humans. This was the result of an interactive process that took place throughout thousands of years and led to competence, cooperation and coevolution relationships. Through a convergent evolution process, dogs have been able to acquire social abilities, similar to those of humans, which enabled particular communication ways between the two species. Dogs' remarkable ability to understand human social and communicative behaviors (even better than genetically related species, such as primates) favored their relationship with humans. Although some debate arose about the accuracy of the concept attachment, evidence of the most recent investigations on behavioral and endocrine responses in human-dog interactions have supported the idea that this relationship represents an expression of attachment. The oxytocin role has stood out in the formation of attachment bonds and in prosocial 
behavior that encourages intraspecies relationships. It achieves this by inducing maternal behaviors in a persistent fashion, influencing on pairing and increasing interpersonal trust through eye contact, empathy, etc. Moreover, the participation of oxytocin in the social bond processes is complex, as it has shown to play multiple roles. Oxytocin influences on the stress by reducing the cortisol levels, modulates the amygdala activity linked to fear, and increases the pain threshold, among other actions. Research in the human-animal interaction field, or Anthrozoology, has highlighted the companion animals' contribution to people wellbeing and particularly the buffering effect on daily life stress. The idea that living with an animal can have a positive influence on human health has been called pet effect and has been studied understanding physiological, psychosocial and therapeutic aspects. We carried out a systematic bibliographic review of the empiric studies about human-dog interactions in which oxytocin levels were assessed or oxytocin was administered, in dogs and / or humans. We present the employed procedures and the main results, and analyze the limitations and relevance of the investigations' findings, as well as their consistencies and contradictions. The results are discussed in function of the findings related to oxytocin and its intraspecies effects, and also in function of the development in Anthrozoology in general. The possible role of the oxytocin in the different aspects of the human-dog relationship is analyzed. Among these aspects we highlight: the pet effect, and the reported benefits of the relationship human-companion dog, mainly related to a decrease in stress symptoms; anthropomorphism and the mental states attribution necessary so that pet keeping makes sense; and the cute response, which implies an instinctive reaction to give parental care as a response to the infantile scheme, thought to be involved in the origin of pet keeping. The oxytocin role as an underlying neuroendocrine substrate that explains the benefits of the positive social experience that implies the human-dog bond and promotes the strengthening of this reciprocate and shared bond is emphasized. Finally, new lines of investigation are considered, such as the empiric study of the relationship of this neurohormone with the anthropomorphism and the response to the infantile scheme. We lay stress on the importance of developing studies with samples that includes humans and dogs of different ages, with the object of assessing the influence of age on the response to oxytocin. We also make a point of the relevance of carrying out comparisons in relation to the human and dog gender, which has been usually controlled rather that investigated. Furthermore, we discuss the validity of the techniques employed to measure and administer the oxytocin. We make recommendations about these techniques and their use for considering the investigations goals. We highlight the least invasive methods such as the urine oxytocin measure, which are related to a more spontaneous behaviors and are more in agreement with animal welfare.

Key words: Anthrozoology; Attachment; Dog; Human-animal bond; Oxytocin.

\section{INTRODUCCIÓN}

Ancestralmente los animales han sido respetados como compañeros esenciales en la supervivencia y en la salud de las personas. Muchas tradiciones espirituales han honrado las relaciones entre humanos y las demás formas de vida animal, como parte de la interconexión con el mundo natural y espiritual (Serpell, 2006). De acuerdo a la evidencia arqueológica y estudios de Biología Molecular, la primera especie en hacer la transición desde un estado salvaje hacia uno doméstico fue el lobo (Canis lupus), antecesor del perro (Canis familiaris). Si bien los fósiles más antiguos de estos últimos tienen 20.000 años de antigüedad, se estima que ese fue sólo el resultado de un proceso interactivo que tuvo lugar durante 80.000 años (Valadez Azúa, 2000), basado en la necesidad compartida de refugio, comida y protección, y que ha dado lugar a relaciones de competencia, cooperación y coevolución entre humanos primitivos y antepasados de los perros (Serpell, 2003). Desde entonces, estos canes han desarrollado una relación especial con los humanos, pudiendo ser considerados como la única especie que ha establecido un nicho propio en la sociedad humana (Nagasawa, Mogi \& Kikusui, 2009). 
Durante la historia compartida con los humanos, los perros han sido sometidos a un intensivo proceso de selección artificial, que ha dado lugar a variaciones morfológicas, genéticas y conductuales (Jakovcevic \& Bentosela, 2009). Hare y Tomasello (2005) destacaron a estos animales como expertos en la lectura de los comportamientos sociales y comunicativos de los humanos (aún más que especies más emparentadas genéticamente, como los primates). Estos autores argumentaron que fue a través de la evolución convergente, que los perros poseyeron habilidades sociales parecidas a las de los humanos, las cuales habilitaron particulares líneas de comunicación entre ambos. Por ejemplo, los perros son capaces de utilizar mirada recíproca como una herramienta comunicativa cuando necesitan ayuda de otros para resolver un problema (Miklósi et al., 2003). Para los perros domésticos, las personas pueden proveer el acceso a recursos valiosos, por lo que una adecuada comunicación con las mismas resulta fundamental (Jakovcevic \& Bentosela, 2012). La mirada desempeña un rol central en la comunicación humana, en tanto facilita el entendimiento de las intenciones del otro, pero además el establecimiento de relaciones de filiación con otros (Nagasawa et al., 2015).

En los últimos años se ha sugerido que la relación humano-perro de compañía podría representar una expresión de apego. Bowlby (1998) desarrolló la teoría del apego para dar cuenta de los mecanismos sobre los cuales la relación padre-hijo influye en el desarrollo del pequeño, a partir de la consideración de aspectos etológicos. Esta teoría se basa en la premisa de que los humanos, como muchos animales, están biológicamente predispuestos para buscar y mantener contacto físico y conexión emocional con figuras selectas a quienes se les confía protección física y psicológica. De este modo, puede dar cuenta de los comportamientos de búsqueda de proximidad como producto de un diseño evolutivo que permite asegurar la proximidad física y disponibilidad emocional de figuras de apego cuando fuera necesaria (Sable, 2013). El apego requiere la distinción de una figura específica utilizando señales sociales particulares de la es- pecie y respuestas particulares a esa figura, provocadas por funciones homeostáticas neuroendocrinas, así como por aspectos comportamentales. Los perros han demostrado que pueden distinguir una figura humana particular y exhibir reacciones autonómicas específicas (Nagasawa et al., 2009).

Recientemente el neuropétido oxitocina atrajo la atención con respecto a las bases neurológicas de los comportamientos prosociales que facilitan las relaciones entre individuos (Romero, Nagasawa, Mogi, Hasegawa $\&$ Kikusui, 2014). Se trata de una hormona nanopeptídica sintetizada en las neuronas de los núcleos supraóptico y paraventricular del hipotálamo. Algunas de estas neuronas proyectan sus axones hacia el lóbulo posterior de la glándula hipófisis y trasladan la oxitocina hasta allí, donde es acumulada para ser luego liberada al sistema circulatorio; otro grupo de neuronas proyecta sus axones directamente a distintas estructuras del cerebro para la liberación de la hormona a nivel local. Por otro lado, se ha detectado también la liberación local de oxitocina a nivel del cuerpo y las terminales dendríticas de estas neuronas hipotalámicas (Landgraf \& Neumann, 2004; Ross et al., 2009).

De manera similar en humanos y otros mamíferos, la oxitocina se encuentra implicada en el apego y comportamientos parentales (Feldman, Gordon \& Zagoory-Sharon, 2011), induce comportamientos maternales de manera persistente (Pedersen, Ascher, Monroe \& Prange, 1982), está implicada en el emparejamiento (Carter, Devries \& Getz, 1995), incrementa la confianza en otras personas (Kosfeld, Heinrichs, Zak, Fischbacher \& Fehr, 2005), incrementa la mirada a los ojos (Guastella, Mitchell \& Dadds, 2008), mejora la habilidad para inferir estados mentales en otros (Domes, Heinrichs, Michel, Berger \& Herpertz, 2007) y la empatía emocional (Hurlemann et al., 2010).

La participación de la oxitocina en los procesos de vinculación social resulta compleja, en tanto ha mostrado tener un rol dual, influyendo sobre los comportamientos de filiación así como también sobre el estrés (Feldman et al., 2011). La oxitocina mostró 
también tener un efecto ansiolítico con reducción de niveles de cortisol (Heinrichs, Baumgartner, Kirschbaum \& Ehlert, 2003), modular la actividad de la amígdala ligada al miedo (Labuschagne et al., 2010) e incrementar los umbrales del dolor y disminuir los niveles de corticosterona (Petersson, Eklund \& UvnäsMoberg, 2005).

La idea de que vivir con un animal puede tener una influencia positiva en la salud humana ha sido llamada efecto mascota (Allen, 2003) y ha sido estudiada comprendiendo aspectos fisiológicos, psicosociales y terapéuticos. La tenencia de mascotas fue relacionada con un mayor índice de supervivencia después de infartos (Friedmann, Katcher, Lynch \& Thomas, 1980), menores niveles de cortisol y triglicéridos (Anderson, Reid \& Jennings, 1992), reducción en los niveles de presión arterial (Allen, Blascovich \& Mendes, 2002), mayor percepción de capacidad y autoeficacia (Zilcha-Mano, Mikulincer \& Shaver, 2012), así como también con el incremento y facilitación de interacciones sociales (Guéguen \& Ciccotti, 2008).

La interacción física entre humanos y perros involucra varios tipos de estimulación sensorial no amenazante, como el contacto, la presión suave, el calor y las caricias, como así también con señales olfativas, auditivas y visuales. En tanto este tipo de estimulación da lugar a una multitud de efectos que pueden en parte, estar mediados por la oxitocina en humanos y en animales, es posible que la liberación de oxitocina y de los efectos mediados por esta sean inducidos durante la interacción entre humanos y perros (Handlin et al., 2011).

\section{ObJetivo}

El objetivo del estudio que se informa fue realizar una revisión sistemática de publicaciones científicas ligadas a la interacción humano-animal y de trabajos empíricos que evalúen los niveles de oxitocina en humanos y / o perros, o bien se administre oxitocina, sobre los cuales elaborar conclusiones acerca del rol desempeñado por esta neurohormona en la relación humano-perro de compañía.

\section{MÉTOdo}

\section{MATERIALES}

La información fue organizada y el diseño de la estructura de revisión se realizó de acuerdo a lo propuesto por Roussos (2011). La búsqueda bibliográfica involucró dos procedimientos básicos: generar un conjunto de artículos potencialmente relevantes y seleccionar una submuestra de artículos y capítulos de libros para incluir en la revisión. Inicialmente se realizaron búsquedas en la Biblioteca Electrónica de Ciencia y Tecnología del Ministerio de Ciencia, Tecnología e Innovación Productiva (MINCYT) que tuvieran alguna de las siguientes combinaciones de palabras claves: oxitocina + perro, oxitocina + mascota, oxitocina + animal de compañía, oxitocina + humano-animal. Todas las búsquedas se realizaron tanto en español como en inglés y los términos utilizados fueron: oxytocin, dog, pet, companion animal, human-animal, en las combinaciones referidas arriba. Estas búsquedas aportaron 236 publicaciones académicas. Los resúmenes fueron revisados y las referencias retenidas sólo cuando evaluaban aspectos ligados a la interacción humano-perro. Esto aportó 32 artículos que fueron descargados y almacenados. Luego se seleccionaron solamente los que eran empíricos y se excluyeron aquellos en los que no se consideraron las concentraciones de oxitocina. Esto aportó 11 artículos que cumplimentaron los criterios de inclusión. Toda la bibliografía seleccionada se encontraba en idioma inglés. En tanto las publicaciones de Odendaal (2000) y Odendaal y Meintjes (2003) se refieren a un mismo estudio empírico, por lo tanto se las consideró como una sola investigación.

\section{DesarRollo}

Los estudios se presentan a continuación y de acuerdo a su fecha de publicación. A su vez, el Cuadro1 muestra algunas de sus principales características.

Odendaal y Meintjes (2003) evaluaron seis neuroquímicos asociados con el descenso de 
la presión arterial en 18 humanos adultos saludables que disfrutaban la interacción con 18 perros antes y después de interacciones positivas (algunos perros pertenecían a los participantes y otros fueron provistos por los experimentadores). Los controles fueron línea de base vs. valores post-interacción, dueños de perros vs. personas interactuando con perros desconocidos e interacción con perro vs. lectura distendida de libros. Las interacciones positivas entre humanos y perros produjeron en ambos un incremento significativo en niveles de $\beta$-endorfinas, oxitocina, prolactina, ácido fenilacético (un metabolito de $\beta$-feniletinamina) y dopamina. El cortisol disminuyó significativamente en humanos, mientras que en perros el descenso no fue significativo. No hubo diferencias significativas entre los cambios en neuroquímicos durante la lectura tranquila y la interacción positiva con los perros, excepto en la oxitocina, prolactina y $\beta$ endorfinas. La oxitocina mostró la única diferencia estadísticamente significativa en medidas pre y post-interacción entre dueño y su propio perro vs. interacción con perro desconocido. Los autores destacaron los cambios en las concentraciones de oxitocina en tanto estas se duplicaron en humanos y perros después de las interacciones positivas entre ambos (Odendaal, 2000; Odendaal \& Meintjes, 2003).

Miller y colaboradores (2009) evaluaron los niveles de oxitocina de 10 hombres y 10 mujeres en el momento previo a entrar en contacto con sus perros, luego de una jornada laboral y 25 minutos más tarde luego de haber estado interactuando con sus animales. Esta condición fue comparada con una condición control en la cual los mismos participantes realizaron una lectura en ausencia de sus perros. En las mujeres luego de haber interactuado con sus perros los niveles de oxitocina se incrementaron un $58.4 \%$, mientras que luego de la lectura de libros se observó una disminución de un $26 \%$ en los niveles de oxitocina. Por otro lado, en hombres no se observaron incrementos en los niveles de oxitocina después de interactuar con sus perros, de hecho, los niveles disminuían luego de ambas condiciones: un $21.5 \%$ luego de la interacción con perro y un $56.3 \%$ luego de la lectura. Los autores sugirieron que hombres y mujeres podrían tener diferentes respuestas hormonales a la interacción con sus perros.

Nagasawa, Kikusui, Onaka y Ohta (2009) evaluaron las concentraciones de oxitocina en orina de un grupo de 55 propietarios (21 de ellos eran hombres) mientras interactuaban con sus perros normalmente durante $30 \mathrm{mi}-$ nutos (interacción experimental), o mientras interactuaban sin mirar directamente a sus perros (control experimental). Los dueños fueron divididos en dos grupos: uno que tendía a recibir miradas de mayor duración de sus perros y que había indicado mayor grado de satisfacción y comunicación con estos (ML) y otro que había recibido miradas de menor duración de sus perros y que había indicado menor grado de relación con estos (MC). El sexo de los perros no mostró diferencias con respecto a esta agrupación. Los niveles de oxitocina resultaron más altos en ML que en MC después de la interacción experimental, pero no después de la situación de control experimental. Los autores concluyeron que las interacciones humano-perro, especialmente aquellas iniciadas a partir de la mirada del perro, podían incrementar las concentraciones urinarias de oxitocina en los dueños, y que estas se relacionarían con comportamientos de apego.

Handlin y colaboradores (2011) evaluaron la frecuencia cardíaca y los niveles de oxitocina, cortisol e insulina de 10 mujeres y sus 10 perros labradores macho en respuesta a interacción a corto plazo. Las dueñas debían acariciar y hablar con sus perros durante los primeros 3 minutos. Las muestras de sangre se tomaron antes de iniciar la interacción (0) y $1,3,5,15,30$ y 60 minutos después. Las respuestas de las dueñas se compararon con las de un grupo control de 10 mujeres que no tenían perros. En los perros, los niveles de oxitocina se incrementaron significativamente a los 3 minutos de iniciada la interacción. Los niveles de cortisol de las dueñas tendían a disminuir a los 15 minutos de haber iniciado la interacción, pero no a los $30 \mathrm{mi}-$ nutos. La frecuencia cardíaca se encontró significativamente disminuida a los 55 y 60 mi- 
nutos en las dueñas, mientras que no se observaron cambios en la frecuencia cardíaca en el grupo control. Tanto las dueñas como los controles experimentaron un descenso de cortisol a lo largo del experimento. Es posible que la situación experimental, que involucra muestras de sangre, haya sido percibida como estresante, lo que pudo haber causado una elevación en los niveles de cortisol de ambos grupos, y luego la disminución gradual a través del tiempo (Handlin et al., 2011). Los autores concluyeron que las interacciones humano-perro producían un incremento en los niveles de oxitocina de ambos, y que en tanto la oxitocina es liberada por estimulación sensorial, la interacción entre perros y dueños puede haber disminuido los niveles de cortisol y frecuencia cardíaca a través de la liberación de oxitocina en el cerebro.

Mitsui y colaboradores (2011) inyectaron oxitocina por vía intravenosa a seis perros labradores para incrementar su concentración en sangre. Determinaron que las concentraciones más altas de oxitocina en orina se daban una hora después de la inyección.

En un segundo experimento evaluaron el efecto de emociones positivas producidas por alimentación, ejercitación y caricias en un grupo de nueve perros. Al grupo control simplemente se le dio de beber agua. Se recolectaron muestras de orina previamente a cada estimulación y posteriormente, según los resultados del experimento anterior. Los resultados mostraron que mientras la condición control no evidenció un incremento en oxitocina, comer, ejercitarse y recibir caricias produjeron un incremento significativo en las concentraciones de oxitocina en orina. A su vez, comer incrementó las concentraciones de cortisol, mientras que los otros estímulos no produjeron efecto en el cortisol.

Handlin, Nilsson, Ejdebäck, HydbringSandberg y Uvnäs-Moberg (2012) con una escala evaluaron el tipo y grado de relación de 10 mujeres con sus 10 perros labradores y correlacionaron los resultados con los niveles de cortisol y oxitocina en sangre de dueñas y perros. Los niveles de oxitocina de las dueñas correlacionaron con cuán a menudo besaban a sus perros. Los niveles de cortisol en las dueñas estuvieron relacionados con sentirse molestas porque el perro les impidiera hacer cosas. Los autores concluyeron que elevados niveles de oxitocina y bajos de cortisol en dueños estaban relacionados con la percepción de estos de la relación con sus perros como placentera, interactiva y asociada con menos problemas. Los niveles más altos de oxitocina en los perros se relacionaban con mayor interacción con sus dueños y con la percepción de los dueños del perro como un compañero positivo y agradable.

Rehn, Handlin, Uvnäs-Moberg y Keeling (2014) evaluaron el comportamiento y los niveles de cortisol y oxitocina en sangre de 12 perras Beagle antes, durante y después del regreso de una persona familiar en tres condiciones diferentes: la persona familiar regresaba e iniciaba contacto físico y verbal con el perro (FV), sólo contacto verbal (V), o la situación control en que la persona ignoraba al perro (C). Los perros eran dejados en una habitación con el técnico veterinario, quien los ignoraba durante 25 minutos. Cada perro fue sometido a las tres condiciones, una por semana en semanas consecutivas, y el orden de presentación de las condiciones fue balanceado. Frente al reencuentro inicialmente se daba un incremento en la oxitocina al ver que la persona se aproximaba. En el tratamiento FV se observó un incremento en los niveles de oxitocina de los perros que se mantuvo aún después de que la interacción había terminado. Si bien los niveles de cortisol mostraron un descenso luego del reencuentro en todas las condiciones, en FV la curva fue más pronunciada, posiblemente como consecuencia de la liberación de oxitocina. En esta condición los perros iniciaban más contacto físico con las personas y tendían a lamer más. Mientras que en el tratamiento $\mathrm{V}$ tendían a mover más la cola y vocalizar, y en el tratamiento $\mathrm{C}$ tendían a redirigir sus conductas de acercamiento hacia el técnico veterinario. Los autores concluyeron que la liberación de oxitocina en FV podría incrementar los comportamientos de acercamiento como el contacto visual y físico iniciado por los perros; en tanto los comportamientos de búsqueda de contacto como las vocalizaciones estuvieron 
más demorados y fueron menos prominentes, es probable que los perros encuentren más reconfortantes los comportamientos anteriores.

Romero y colaboradores (2014) aplicaron oxitocina intranasal a 16 perros (8 eran machos) y evaluaron sus comportamientos para con sus dueños y con otro perro conocido. Al grupo control se le aplicó solución salina. Se realizaron además mediciones de oxitocina en sangre y orina. La oxitocina exógena promovió comportamientos sociales positivos tanto hacia sus conespecíficos como hacia los humanos. Los perros del grupo experimental mostraron mayor orientación social y filiación hacia sus dueños, y mayor filiación y comportamientos de aproximación hacia sus perros compañeros, en comparación con el grupo placebo. Machos y hembras no mostraron diferencias en cuanto a comportamientos de filiación hacia conespecíficos. Los comportamientos de intercambios sociales positivos con los otros perros dispararon la liberación de oxitocina endógena, indicando la estimulación del sistema de oxitocina durante las interacciones sociales. Los autores concluyeron teorizando sobre la existencia de un bucle de retroalimentación positiva mediatizado por la oxitocina en la vinculación de los perros.

Oliva, Rault, Appleton y Lill (2015) administraron oxitocina intranasal a 62 perros (31 de ellos eran machos) para evaluar su efecto sobre su cognición social. Los perros completaron el experimento en dos sesiones con 5-15 días de separación, en las cuales recibían uno de los dos tratamientos: oxitocina o solución salina. En ambas sesiones los perros debían realizar dos series de pruebas: una que implicaba utilizar una pista de señalamiento distal breve y la otra que implicaba una señal con la mirada. Se implementó también una condición de control en la que el experimentador no brindaba pista alguna. El grupo que recibió la aplicación de oxitocina en la primera sesión obtuvo un mejor rendimiento frente al señalamiento distal breve. Con respecto a las pruebas de señal con la mirada bajo la administración salina, los perros obtuvieron un puntaje por debajo del azar, mientras que tras la aplicación de oxi- tocina los perros obtuvieron un mejor rendimiento equiparando el azar. Los autores plantearon la posibilidad de que las respuestas podían deberse a cierta aversión de los perros frente a la señalización a través de la mirada, y que entonces la oxitocina pudo haber tenido un efecto inhibitorio sobre esta aversión.

Nagasawa y colaboradores (2015) reelaboraron su investigación anterior complejizando el experimento. En una primera parte recolectaron muestra de orina antes y $30 \mathrm{mi}-$ nutos después de la interacción entre parejas de humanos y cánidos (perros o lobos criados por humanos). Analizaron las interacciones (mirada-del-perro, mirada-del-dueño y tocar-al-perro) y dividieron a los dueños de perros nuevamente en grupos de $\operatorname{ML}(n=21)$ y MC $(n=9)$. Realizaron comparaciones en interacciones y niveles de oxitocina entre estos y con el grupo de 11 dueños de lobos. Sólo los dueños del grupo ML mostraron un incremento significativo en las concentraciones de oxitocina y el mayor cambio proporcional de oxitocina. Luego de un análisis de regresión lineal múltiple encontraron que sólo la duración de la mirada-del-perro explicaba significativamente el cambio proporcional de oxitocina en el dueño. La duración de tocar-al-perro evidenció una tendencia a explicar las concentraciones de oxitocina en los dueños. Los perros del grupo ML tuvieron un incremento significativamente mayor de oxitocina que los del grupo MC. La duración de la mirada-del-perro explicó significativamente el cambio proporcional de oxitocina en los perros y la duración de tocar-alperro mostró una tendencia a explicar las concentraciones de oxitocina en los perros. Los lobos extrañamente mostraron mirada recíproca con sus dueños y su mirada hacia los dueños no correlacionó con cambios en oxitocina en dueños ni lobos, ni explicó el cambio proporcional en lobos ni dueños. En este experimento no se observaron diferencias de acuerdo al sexo del animal.

En un segundo experimento se administró una solución con oxitocina o salina a un grupo de 27 perros antes de ingresar a una sala experimental donde su dueño y dos personas 
desconocidas debían permanecer sentadas limitando sus interacciones. Se analizaron muestras de orina de dueño y perro previo y posterior a la interacción y se codificaron los comportamientos del perro (mirada-del-perro, contacto y la proximidad al dueño y los desconocidos). Los investigadores encontraron que la administración de oxitocina incrementaba la mirada-del-perro (sólo en hembras), lo cual daba lugar a un incremento en los niveles de oxitocina de los dueños. Los niveles de oxitocina en perros no mostraron diferencias, posiblemente debido a la limitación de la interacción de los dueños, quienes no podían tocar voluntariamente al perro.

\section{DISCUSIÓN}

En los experimentos de Handlin y colaboradores (2011) y Odendaal y Meintjes (2003) se observó que los niveles de cortisol tendían a disminuir en los participantes humanos luego de interactuar con sus perros, al mismo tiempo que los niveles de oxitocina tendían a incrementarse. Por su parte, en el experimento de Mitsui y colaboradores (2011) comer incrementó los niveles de cortisol en perros, mientras que ejercitarse y recibir caricias no, siendo que se reconoce generalmente que comer y ejercitarse incrementan el cortisol. Para los autores, el incremento de la oxitocina durante el ejercicio pudo haber inhibido el cortisol en orina. Contrariamente, Odendaal y Meintjes (2003) encontraron que a pesar del incremento de oxitocina plasmática en perros, el cortisol tendía a incrementar posteriormente la interacción. Handlin y colaboradores (2011) observaron también un incremento en niveles de cortisol, que luego tendía a disminuir y podría estar reflejando la actividad física dada en la interacción o bien el estrés ligado a la situación experimental. Estos resultados ponen de manifiesto que el efecto inhibitorio de la oxitocina en la secreción de cortisol en perros puede no siempre ser observado y requiere mayor investigación.

Es posible también que sea importante analizar más detenidamente las diferencias en función del sexo del perro. Por ejemplo, Oli- va y colaboradores (2015) indicaron que tanto machos como hembras tuvieron un desempeño similar en una tarea de elección de objeto luego de la administración de oxitocina, pero que los machos incrementaron ligeramente su desempeño bajo la aplicación de solución salina, mientras que las hembras declinaron su desempeño en esta condición. Nagasawa y colaboradores (2015) no observaron diferencias al evaluar los niveles de oxitocina en la interacción, pero sí lo hicieron tras la aplicación de oxitocina intranasal. Para estos autores estas diferencias, también observadas en humanos (Rilling et al., 2014), pueden deberse a que las hembras resulten más sensibles a los efectos de la oxitocina sobre la filiación o que la oxitocina exógena pueda también estar activando el sistema de receptores de vasopresina preferencialmente en machos. Estos podrían haber sido influidos por la presencia de las personas desconocidas mostrando comportamientos de mayor vigilancia, lo cual destaca la complejidad de la oxitocina con respecto a los roles sociales y contextos, también en perros.

Los comportamientos implicados en las interacciones no fueron considerados por Odendaal y Meintjes (2003) ni por Miller y colaboradores (2009). Handlin y colaboradores (2012) encontraron que quienes indicaban besar más a menudo a su perro, tenían niveles de oxitocina más elevados. Por su parte, Rehn y colaboradores (2014) resaltaron la importancia del contacto físico con el perro, contra la mera presencia de la persona o el hecho de que esta le hablara, con respecto a los efectos endócrinos causados por la interacción. Nagasawa, Kikusui y colaboradores (2009) realizaron el primer estudio que investigó la reacción del sistema neuroendócrino humano ante señales sociales de otras especies. La mirada y el contacto del perro se correlacionaron con un aumento de oxitocina en quienes recibían más miradas del perro, mientras que no fue así en quienes recibían menos. Este estudio sugirió que de todas las interacciones, el sostenimiento de la mirada del perro, un factor que contribuye a los lazos sociales, tenía un efecto particularmente intenso en el sistema neuroendócrino del dueño. 
El rol de la mirada y su relación con la oxitocina ya había sido destacado en humanos. Un experimento mostró cómo la aplicación intranasal de oxitocina incrementaba la mirada hacia los rostros, específicamente hacia la región de los ojos (Guastella et al., 2008). Los autores identificaron a esto como el mecanismo por el cual la oxitocina mejora el reconocimiento de emociones, la comunicación interpersonal y los comportamientos de acercamiento social en humanos.

Posteriormente, Nagasawa y colaboradores (2015) volvieron a destacar la importancia de la mirada del perro en la liberación de oxitocina en el dueño. En este estudio, además, al evidenciar que los lobos no utilizaban la mirada como forma de comunicación social con humanos, sugirieron que la mirada del perro al dueño habría evolucionado como una forma de comunicación social durante la domesticación y que dispararía la liberación de oxitocina en el dueño, facilitando la interacción mutua y la comunicación de filiación y consecuentemente la activación del sistema de oxitocina tanto en humanos como en perros en un bucle positivo.

Aunque tempranamente los resultados de Odendaal y Meintjes (2003) sugirieron un rol para la oxitocina en el mantenimiento de asociaciones cooperativas, fallaron en demostrar que la oxitocina inducía cambios en el comportamiento que facilitarían esa relación. Algo similar sucedió con el estudio de Handlin y colaboradores (2012) debido a la naturaleza correlacional de su estudio. Romero y colaboradores (2014) mostraron que la administración de oxitocina a los perros promovía los comportamientos sociales positivos hacia sus conespecíficos y que estos disparaban la liberación de oxitocina endógena. Los autores indicaron que la oxitocina mediaría un bucle de retroalimentación positiva en la vinculación social de los perros. Estos datos aportaron información sobre los mecanismos que facilitan el mantenimiento de relaciones sociales cercanas más allá del interés reproductivo inmediato o de los lazos genéticos. En tanto la oxitocina aumenta la recompensa a través de las vías mesolímbicas dopamino-dependientes, es posible que un feedback positivo, a través de la inducción de secreción de oxitocina por el comportamiento, incentive interacciones repetidas con individuos con quienes han ocurrido interacciones positivas previamente (Donaldson \& Young, 2008; Romero et al., 2014; Swain et al., 2014). Los resultados de la investigación de Nagasawa y colaboradores (2015) apoyaron la existencia de un bucle interespecies mediado por la oxitocina, facilitado y modulado por la mirada, lo cual puede haber apoyado la coevolución del vínculo humano-perro a través del empleo de formas comunes de comunicación y apoyo social.

En los resultados de Nagasawa, Kikusui y colaboradores (2009), la presentación de los dueños como estímulo activaba el sistema autonómico de los perros más que cuando se presentaban extraños. Esto evidenció la habilidad de los perros para discriminar y responder diferencialmente a humanos distintos, cumplimentando algunos de los criterios para la formación de un vínculo social entre humanos y perros. Handlin y colaboradores (2012) destacaron la relación positiva entre los niveles de oxitocina de perros y dueños como un indicador de la existencia de una relación mutua. De un modo similar, Romero y colaboradores (2014) encontraron que el incremento en los niveles de oxitocina estaba asociado con que la filiación tenga reciprocidad, más que con el solo hecho de proveerla o recibirla. Estos parámetros darían cuenta de la existencia de un vínculo, recíproco y compartido, humano-perro. Nagasawa, Kikusui y colaboradores (2009) también destacaron la existencia de una relación entre los niveles de oxitocina y el apego al animal. Para estos autores, un dueño que es contemplado por su perro percibe una condición emocional del perro y considera la mirada como un comportamiento de apego. El resultado de que las concentraciones de oxitocina eran afectadas si los perros o los dueños iniciaban el intercambio indicaría que la mirada de los perros cumple los requerimientos para un comportamiento de apego.

Que los humanos respondan a las señales relacionadas con el apego de otras especies puede ser explicado por la naturaleza humana 
y la tendencia al antropomorfismo. De modo similar al comportamiento observado frente a infantes humanos, al ser mirado por su perro el dueño puede especular acerca del estado emocional del perro y considerar esa mirada como un comportamiento de apego (Nagasawa, Mogi et al., 2009).

Que la oxitocina exógena promueva un conjunto de comportamientos directamente relacionados con la vinculación social en perros, apoya la idea de que los mismos factores hormonales y neuroendócrinos que promueven comportamientos parentales y emparejamiento en mamíferos (i.e., el sistema oxitocinérgico) también contribuyen a la formación de otros tipos de relaciones sociales (Romero et al., 2014). Los mecanismos neurohormonales que evolucionaron para facilitar las relaciones sociales con otros humanos serían la base para la formación del apego emocional que enlaza a los humanos con otras especies (Herzog, 2014).

Se ha destacado que las características del esquema infantil, compartidas por crías de animales y humanos, funcionarían como liberadores de instintos maternales porque automáticamente hacen aflorar impulsos parentales (Herzog, 2012). A esta atracción humana innata hacia todo lo que tenga apariencia de cría se le llamó respuesta a lo adorable (cute response - Lorenz, 1943) y se la ha vinculado al origen de la tenencia de mascotas. Algunos autores han sugerido que la tenencia de animales de compañía puede haberse originado en la era paleolítica como consecuencia de un comportamiento parental mal dirigido, pudiendo entenderse como una forma de adopción cruzada de especies (Serpell, 2003; Serpell \& Paul, 2011). Es posible que el bucle interespecies mediado por la oxitocina haya desempeñado un rol importante en este proceso. De hecho, diversos estudios evidenciaron el efecto de la oxitocina en la aloparentalidad (e.g., Pedersen et al., 1982).

Existe una perspectiva evolutiva que tiende a considerar la tenencia de animales de compañía como resultado de un proceso de parasitación social (e.g., Archer, 1997), desde la cual podría resultar tentador buscar respaldo en el sistema de activación de oxitocina.
Que la oxitocina promueva la aproximación social y afiliación en otras relaciones que madre-hijo o parejas no debe interpretarse como un efecto indiscriminado de la oxitocina en las relaciones sociales. El efecto de la oxitocina sobre el comportamiento y la cognición social depende del contexto. Por ejemplo en humanos y otros animales, la oxitocina puede incrementar la filiación y cooperación o incrementar la agresividad para proteger la descendencia y los vínculos de pareja (Campbell, 2008). Se demostró también que la oxitocina incrementa los comportamientos prosociales, pero sólo en ausencia de señales de que el otro sea indigno de confianza (Mikolajczak et al., 2010).

Otra característica que ha sido relacionada con el proceso de asimilación de las primeras mascotas a las familias humanas, se refiere al antropomorfismo, el cual resulta un rasgo casi universal entre los tenedores de mascotas (Serpell, 2003; Serpell \& Paul, 2011). Es posible que esta capacidad interpretativa-atributiva que resulta indispensable para la interacción social humana también lo sea para el establecimiento de una relación humano-perro y que se vea favorecida por la activación del sistema de oxitocina. Domes y colaboradores (2007) mostraron que la oxitocina mejoraba la habilidad humana para leer la mente a otros individuos, esto es, para inferir estados mentales a través de la interpretación de pistas sociales sutiles. Recientemente otro estudio evidenció que la administración de oxitocina incrementa la tendencia humana al antropomorfismo (Scheele et al., 2015). Serpell (2003) conjeturó previamente que el antropomorfismo habría permitido utilizar a los animales como fuentes alternativas de apoyo social habilitando los recursos para beneficiarse emocional y físicamente con esto.

La oxitocina también ha sido sugerida como la molécula candidata para la transmisión de los efectos de amortiguación social (Nagasawa, Mogi et al., 2009). Heinrichs y colaboradores (2003) mostraron que si bien la oxitocina tenía un efecto ansiolítico, los participantes de su experimento que habían recibido oxitocina y apoyo social de un amigo, y no sólo uno de estos tratamientos, eran quie- 
nes contaban con las menores concentraciones de cortisol, incremento en su tranquilidad y disminución de la ansiedad frente a estresores. Así, la oxitocina mejoraría el efecto de amortiguación del apoyo social en respuesta al estrés.

Una de las teorías mejor sustentadas para dar cuenta del efecto mascota plantea que algunos de los beneficios en la salud humana, conferidos por estos animales son derivados de su rol como proveedores de apoyo social, y que este apoyo actúa como un amortiguador contra el estrés de la vida diaria (Allen et al., 2002). Que los perros (y posiblemente otros animales de compañía) resulten sumamente efectivos al momento de reducir los efectos del estrés, podría deberse a su configuración como fuentes de apoyo social a través de interacciones que a su vez favorecen la secreción de oxitocina, la cual mejora el efecto de amortiguación.

\section{Conclusiones}

Los mismos mecanismos neurohormonales implicados en la regulación de vínculos intraespecíficos en mamíferos parecen subyacer a la formación de filiaciones estables entre individuos de distintas especies, y la oxitocina puede ser destacada como un neuropéptido clave en los procesos de vinculación social.

Si bien la oxitocina tiene efecto sobre la sintomatología ligada a la ansiedad social, así como también incrementa la percepción de las señales sociales, los resultados de las investigaciones se orientan más bien a destacar a la oxitocina como una promotora de la motivación para la filiación en su efecto sobre el comportamiento social.

A su vez, la oxitocina estaría implicada en dos mecanismos que han favorecido originalmente y continúan favoreciendo, la adopción de animales a las familias humanas: las respuestas paternales frente a las características infantiles y el antropomorfismo.

El repertorio de comportamientos evidenciado por los perros en las interacciones con humanos (búsqueda de contacto físico y tendencia a lamer, reforzada con movimientos de cola más marcados y vocalizaciones) y las respuestas endócrinas evidenciadas en ambos, resultan fundamentales para el mantenimiento de un vínculo, y a su vez el contacto físico recíproco no amenazante entre perros y humanos tiene efectos beneficiosos y tranquilizantes para ambas especies.

Las interacciones sociales positivas han sido relacionadas con efectos promotores de salud y la liberación de oxitocina en respuesta a estímulos sociales, parecen ser parte del sustrato neuroendócrino que subyace a los beneficios de las experiencias sociales positivas a la vez que fomenta el estrechamiento de los vínculos, sea que estos se desarrollen entre humanos, o bien, entre 'especies compañeras', como es el caso de humanos y perros.

\section{RECOMENDACIONES Y FUTURAS LÍNEAS DE INVESTI- GACIÓN}

La asociación planteada entre los niveles de oxitocina y la tendencia al antropomorfismo de los perros de compañía, así como entre estos niveles y las respuestas a las características del esquema infantil de los perros carece por el momento, hasta donde los autores tienen conocimiento, de investigaciones empíricas que las fundamenten. La tendencia al antropomorfismo ha sido evaluada a partir de inventarios (e.g., Boya, Dotson \& Hyatt, 2012; Dotson \& Hyatt, 2008) y las respuestas a las características juveniles o neoténicas, a través de la puntuación del grado de atracción hacia determinadas fotografías de rostros humanos y animales (e.g., Archer \& Monton, 2011; Sprengelmeyer et al., 2009).

La medición de los niveles de oxitocina en respuesta a la interacción con perros adultos en comparación con cachorros también puede resultar útil al momento de evaluar el sustrato de la respuesta a lo adorable, así como la influencia de estas características infantiles en los niveles de oxitocina y la necesidad de controlar la edad y el fenotipo de los perros en los estudios sobre esta neurohormona.

Otra cuestión que demanda mayor investigación con respecto a las respuestas neuro- 
endócrinas en interacciones humano-perro, se relaciona con las posibles diferencias de grupos en función del sexo de los humanos como de los perros, así como el estado reproductivo de estos últimos. Las investigaciones han tendido mayormente a controlar las diferencias antes que a indagarlas.

El método de medición de oxitocina debe ser elegido cuidadosamente considerando los objetivos de la investigación. Aunque pueden resultar procedimentalmente más simples, las mediciones periféricas de oxitocina han sido cuestionadas; pero, además, parece fundamental que los procedimientos no incluyan comparaciones entre distintos métodos de medición de oxitocina, las cuales pueden no ser válidas (Beetz, Uvnäs-Moberg, Julius \& Kotrschal, 2012). Por ejemplo, algunas investigaciones mostraron que las concentraciones de oxitocina en sangre y orina podrían no estar relacionadas (e.g., Feldman et al., 2011). La relación entre niveles plasmáticos de oxitocina, que refleja principalmente la oxitocina liberada por la glándula pituitaria, y la oxitocina detectada en la orina todavía no es clara, así como tampoco resulta tan claro si la oxitocina medida periféricamente retroalimenta la del cerebro (Romero et al., 2014; Uvnäs-Moberg, Handlin \& Petersson, 2011).
A su vez, a la aplicación intranasal de oxitocina se le ha cuestionado su capacidad de atravesar la barrera hematoencefálica y ejercer influencia directa en el cerebro; por lo que puede ser recomendable la administración de oxitocina endovenosa, siempre que sea controlado el estresor que una inyección representa para los sujetos.

También es conveniente considerar los momentos elegidos para realizar la recolección de muestras para las mediciones. Mitsui y colaboradores (2011) indicaron que las concentraciones más altas de oxitocina en orina se habían dado una hora después de la primera inyección de oxitocina. También es relevante la cantidad de muestras tomadas en tanto la oxitocina es liberada en pulsos, por lo que un tiempo corto de muestreo es más probable que refleje los picos más claramente.

Otro aspecto a considerar en los procedimientos es que los métodos no invasivos, como la medición de oxitocina en orina se asocian con comportamientos que ocurren espontáneamente y pueden reflejar más fielmente los cambios, evitando alteraciones en los resultados esperables para situaciones en las que se desarrollen reiteradas mediciones en sangre con tiempos cortos de muestreo. Además de ser más acordes a la perspectiva del bienestar animal. 


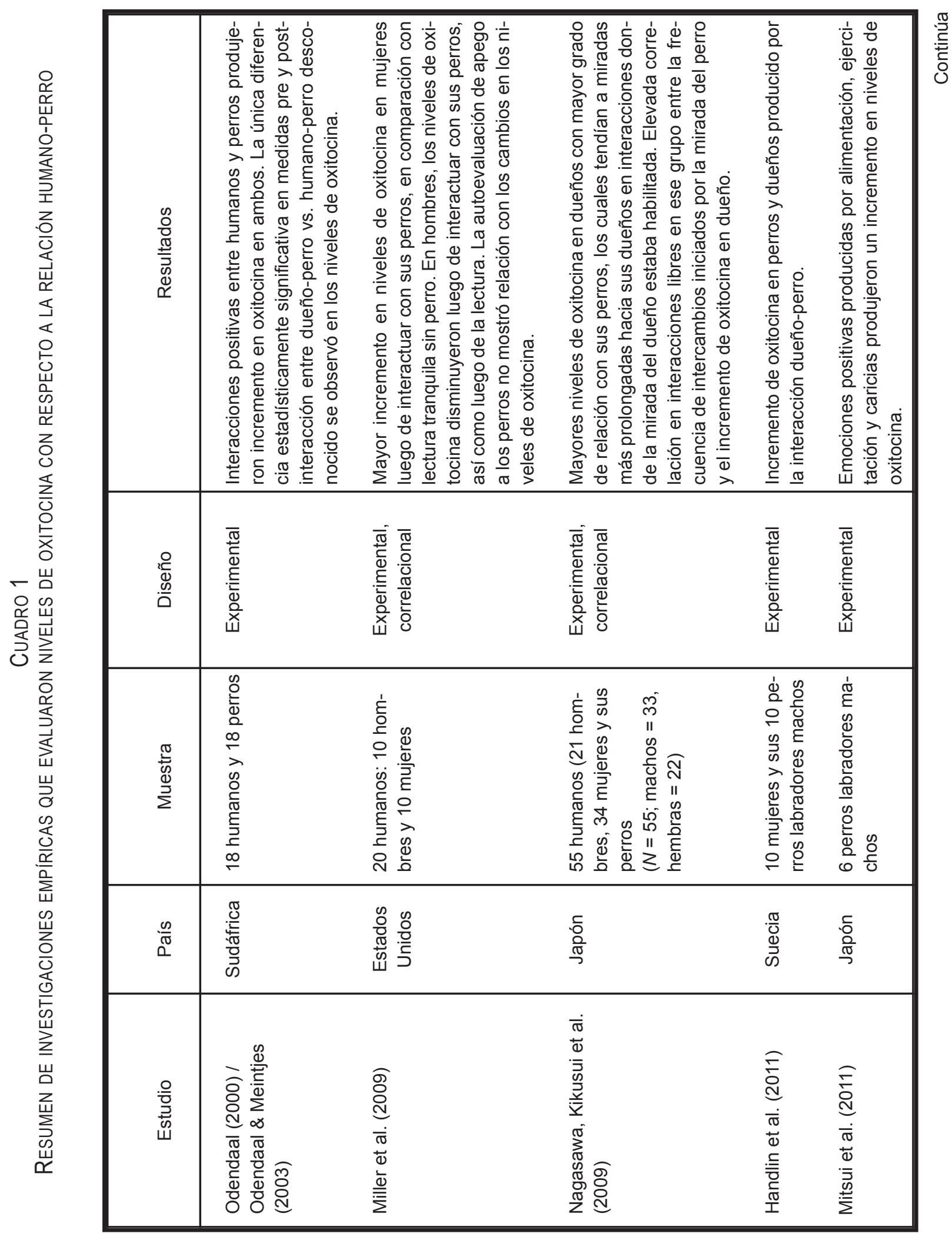




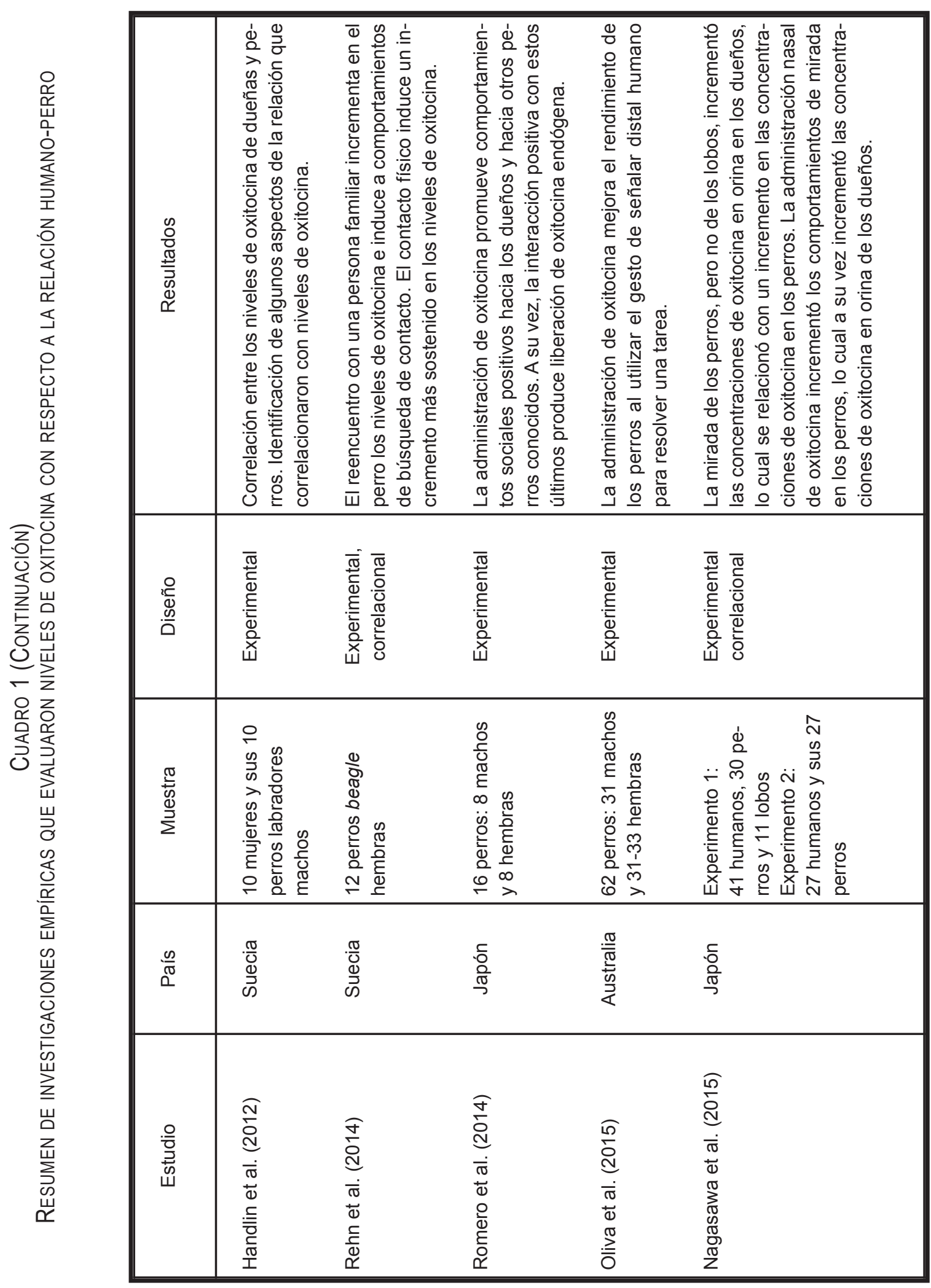




\section{REFERENCIAS BIBLIOGRÁFICAS}

Allen, K. (2003). Are pets a healthy pleasure? The influence of pets on blood pressure. Current Directions in Psychological Science, 12(6), 236-239. http://doi.org/10.1046/j.0963-7214.20 03.01269.x

Allen, K., Blascovich, J. \& Mendes, W.B. (2002). Cardiovascular reactivity and the presence of pets, friends, and spouses: The truth about cats and dogs. Psychosomatic Medicine, 64, 727739 .

Anderson, W.P., Reid, C.M. \& Jennings, G.L. (1992). Pet ownership and risk factors for cardiovascular disease. The Medical Journal of Australia, 157(5), 298-301.

Archer, J. (1997). Why do people love their pets? Evolution and Human Behavior, 18(4), 237259. http://doi.org/10.1016/S0162-3095(99)80 001-4

Archer, J. \& Monton, S. (2011). Preferences for infant facial features in pet dogs and cats. Ethology, 117(3), 217-226. http://doi.org/10.1111/ j.1439-0310.2010.01863.x

Beetz, A., Uvnäs-Moberg, K., Julius, H. \& Kotrschal, K. (2012). Psychosocial and psychophysiological effects of human-animal interactions: The possible role of oxytocin. Frontiers in Psychology, 3(234). http://doi.org/10.3389/ fpsyg.2012.00234

Bowlby, J. (1998). El apego y la pérdida 1: El apego [Attachment and loss 1: Attachment]. Barcelona: Paidós.

Boya, U.O., Dotson, M.J. \& Hyatt, E.M. (2012). Dimensions of the dog-human relationship: A segmentation approach. Journal of Targeting, Measurement and Analysis for Marketing, 20 (2), 133-143. http://doi.org/10.1057/jt.2012.8

Campbell, A. (2008). Attachment, aggression and affiliation: The role of oxytocin in female social behavior. Biological psychology, 77(1), 1-10. http://doi.org/10.1016/j.biopsycho.2007.09.001

Carter, C.S., Devries, A.C. \& Getz, L.L. (1995). Physiological substrates of mammalian mono- gamy: The prairie vole model. Neuroscience \& Biobehavioral Reviews, 19(2), 303-314. http:// doi.org/10.1016/0149-7634(94)00070-H

Domes, G., Heinrichs, M., Michel, A., Berger, C. \& Herpertz, S.C. (2007). Oxytocin improves "mind-reading" in humans. Biological Psychiatry, 61(6), 731-733. http://doi.org/10.1016/j.b iopsych.2006.07.015

Donaldson, Z.R. \& Young, L.J. (2008). Oxytocin, vasopressin, and the neurogenetics of sociality. Science, 322(5903), 900-904. http://doi.org/10. 1126/science. 1158668

Dotson, M.J. \& Hyatt, E.M. (2008). Understanding dog-human companionship. Journal of Business Research, 61(5), 457-466. http://doi.org/ 10.1016/j.jbusres.2007.07.019

Feldman, R., Gordon, I. \& Zagoory-Sharon, O. (2011). Maternal and paternal plasma, salivary, and urinary oxytocin and parent-infant synchrony: Considering stress and affiliation components of human bonding. Developmental Science, 14(4), 752-761. http://doi.org/10.1111 j.1467-7687.2010.01021.x

Friedmann, E., Katcher, A.H., Lynch, J.J. \& Thomas, S.A. (1980). Animal companions and oneyear survival of patients after discharge from a coronary care unit. Public Health Reports, 95 (4), 307.

Guastella, A.J., Mitchell, P.B. \& Dadds, M.R. (2008). Oxytocin increases gaze to the eye region of human faces. Biological Psychiatry, 63(1), 3-5. http://doi.org/10.1016/j.biopsych. 2007.06.026

Guéguen, N. \& Ciccotti, S. (2008). Domestic dogs as facilitators in social interaction: An evaluation of helping and courtship behaviors. Anthrozoos: A Multidisciplinary Journal of the Interactions of People \& Animals, 21(4), 339-349. http://doi. org/10.2752/175303708X3715 64

Handlin, L., Hydbring-Sandberg, E., Nilsson, A., Ejdebäck, M., Jansson, A. \& Uvnäs-Moberg, K. (2011). Short-term interaction between dogs and their owners: Effects on oxytocin, cortisol, insulin and heart rate-an exploratory study. 
Anthrozoös, 24(3), 301-315. http://doi.org/10. 2752/175303711X13045914865385

Handlin, L., Nilsson, A., Ejdebäck, M., HydbringSandberg, E. \& Uvnäs-Moberg, K. (2012). Associations between the psychological characteristics of the human-dog relationship and oxytocin and cortisol levels. Anthrozoös, 25(2), 215-228. http://doi.org/10.2752/ 175303712X1 3316289505468

Hare, B. \& Tomasello, M. (2005). Human-like social skills in dogs? Trends in Cognitive Sciences, 9(9), 439-444. http://doi.org/10.1016/j. tics.2005.07.003

Heinrichs, M., Baumgartner, T., Kirschbaum, C. \& Ehlert, U. (2003). Social support and oxytocin interact to suppress cortisol and subjective responses to psychosocial stress. Biological Psychiatry, 54(12), 1389-1398. http://doi.org/10.10 16/S0006-3223(03)00465-7

Herzog, H. (2012). Los amamos, los odiamos y... los comemos: Esa relación tan especial con los animales [Some we love, some we hate, some we eat: Why it's so hard to think straight about animals]. Barcelona: Editorial Kairós.

Herzog, H. (2014). Biology, culture, and the origins of pet-keeping. Animal Behavior and Cognition, 1(3), 296-308. http://doi.org/10.12 966/ab c.08.06.2014

Hurlemann, R., Patin, A., Onur, O.A., Cohen, M.X., Baumgartner, T., Metzler, S. et al. (2010). Oxytocin enhances amygdala-dependent, socially reinforced learning and emotional empathy in humans. The Journal of Neuroscience, 30(14), 4999-5007. http://doi.org/10.1523/JNE UROSCI.5538-09.2 010

Jakovcevic, A. \& Bentosela, M. (2009). Diferencias individuales en los perros domésticos $(\mathrm{Ca}$ nis familiaris): Revisión de las evaluaciones conductuales [Individual differences in domestic dogs (Canis familiaris): A behavioral assessment review]. Interdisciplinaria, 26(1), 49-76.

Jakovcevic, A. \& Bentosela, M. (2012). Mira lo que te muestro: ¿Comunicación referencial entre perros domésticos (Canis familiaris) y hu- manos? [Look what I show you: Referential communication between domestic dog (canis familiaris) and humans?] Interdisciplinaria, 29 (1), 5-22. https://doi.org/10.16888/interd.2012. 29.1.1

Kosfeld, M., Heinrichs, M., Zak, P. J., Fischbacher, U. \& Fehr, E. (2005). Oxytocin increases trust in humans. Nature, 435(7042), 673-676. http:// doi.org/10.1038/nature03701

Labuschagne, I., Phan, K.L., Wood, A., Angstadt, M., Chua, P. et al. (2010). Oxytocin attenuates amygdala reactivity to fear in generalized social anxiety disorder. Neuropsychopharmacology, 35 (12), 2403-2413. http://doi.org/10.1038/npp.20 10.123

Landgraf, R. \& Neumann, I.D. (2004). Vasopressin and oxytocin release within the brain: A dynamic concept of multiple and variable modes of neuropeptide communication. Frontiers in $\mathrm{Neu}$ roendocrinology, 25(3), 150-176. http://doi.org/ 10. 1016/j.yfrne.2004.05.001

Lorenz, K. (1943). Die angeborenen Formen möglicher Erfahrung [The congenital forms of possible experience]. Zeitschrift für Tierpsychologie, 5, 235-409.

Miklósi, Á., Kubinyi, E., Topál, J., Gácsi, M., Virányi, Z. \& Csányi, V. (2003). A simple reason for a big difference: Wolves do not look back at humans, but dogs do. Current Biology, 13(9), 763-766. http://doi.org/10.1016/S09609822(03)00263-X

Mikolajczak, M., Gross, J.J., Lane, A., Corneille, O., de Timary, P. \& Luminet, O. (2010). Oxytocin makes people trusting, not gullible. Psychological Science, 21(8), 1072-1074. http:// doi.org/10.1177/0956797610377343

Miller, S.C., Kennedy, C.C., DeVoe, D.C., Hickey, M., Nelson, T. \& Kogan, L. (2009). An examination of changes in oxytocin levels in men and women before and after interaction with a bonded dog. Anthrozoös, 22(1), 31-42. http:// doi.org/10.2752/175303708X390455

Mitsui, S., Yamamoto, M., Nagasawa, M., Mogi, K., Kikusui, T., Ohtani, N. \& Ohta, M. (2011). 
Urinary oxytocin as a noninvasive biomarker of positive emotion in dogs. Hormones and Behavior, 60(3), 239-243. http://doi.org/10.101 6/j.yhbeh.2011.05.012

Nagasawa, M., Kikusui, T., Onaka, T. \& Ohta, M. (2009). Dog's gaze at its owner increases owner's urinary oxytocin during social interaction. Hormones and Behavior, 55(3), 434-441. http://doi.org/10.1016/j. yhbeh.2008.12.0 02

Nagasawa, M., Mitsui, S., En, S., Ohtani, N., Ohta, M., Sakuma, Y. et al. (2015). Oxytocin-gaze positive loop and the coevolution of human-dog bonds. Science, 348(6232), 333-336. http://doi. org/10.1126/ science. 1261022

Nagasawa, M., Mogi, K. \& Kikusui, T. (2009). Attachment between humans and dogs. Japanese Psychological Research, 51(3), 209-221. http://doi.org/10.1111/j.1468-5884.2009.0040 2.x

Odendaal, J.S.J. (2000). Animal-assisted therapymagic or medicine? Journal of Psychosomatic Research, 49(4), 275-280. http://doi.org/10.1 016/S0 022-3999(00)00183-5

Odendaal, J.S. \& Meintjes, R.A. (2003). Neurophysiological correlates of affiliative behaviour between humans and dogs. The Veterinary Journal, 165(3), 296-301. http://doi.org/10.1016/ S1090-0233(02)00237-X

Oliva, J.L., Rault, J.L., Appleton, B. \& Lill, A. (2015). Oxytocin enhances the appropriate use of human social cues by the domestic dog (Canis familiaris) in an object choice task. Animal Cognition, 18(3), 767-775. http://doi. org/10.1007/s10071-015-0843-7

Pedersen, C.A., Ascher, J.A., Monroe, Y.L. \& Prange, A.J. (1982). Oxytocin induces maternal behavior in virgin female rats. Science, 216 (4546), 648-650. http://doi.org/10.1126/scienc e. 7071605

Petersson, M., Eklund, M. \& Uvnäs-Moberg, K. (2005). Oxytocin decreases corticosterone and nociception and increases motor activity in OVX rats. Maturitas, 51(4), 426-433. http://doi. org/10.1016/j.maturitas.2004.10.005
Rehn, T., Handlin, L., Uvnäs-Moberg, K. \& Keeling, L.J. (2014). Dogs' endocrine and behavioural responses at reunion are affected by how the human initiates contact. Physiology \& Behavior, 124, 45-53. http://doi.org/10.1016/j. physbeh.2013.10.009

Rilling, J.K., DeMarco, A.C., Hackett, P.D., Chen, X., Gautam, P., Stair, S. et al. (2014). Sex differences in the neural and behavioral response to intranasal oxytocin and vasopressin during human social interaction. Psychoneuroendocrinology, 39, 237-248. http://doi.org/10. 1016/ j.psyneuen.2013.09.022

Romero, T., Nagasawa, M., Mogi, K., Hasegawa, T. \& Kikusui, T. (2014). Oxytocin promotes social bonding in dogs. Proceedings of the National Academy of Sciences, 111(25), 90859090. http://doi.org/10.1073/pnas.1322868111

Romero, T., Nagasawa, M., Mogi, K., Hasegawa, T. \& Kikusui, T. (2015). Intranasal administration of oxytocin promotes social play in domestic dogs. Communicative \& Integrative Biology, 8(3), e1017157. http://doi.org/10.108 0/194 20889.2015.1017157

Ross, H.E., Cole, C.D., Smith, Y., Neumann, I.D., Landgraf, R., Murphy, A.Z. \& Young, L.J. (2009). Characterization of the oxytocin system regulating affiliative behavior in female prairie voles. Neuroscience, 162(4), 892-903. http:// doi.org/10.1016/j.neuroscience.2009.05.055

Roussos, A.J. (2011). Preparación de una revisión bibliográfica para su publicación cuando un solo artículo nos habla de muchos trabajos [Preparation of a literature review for publication when a single article talks about many jobs]. Reportes de Investigación, 1, 1-7.

Sable, P. (2013). The pet connection: An attachment perspective. Clinical Social Work Journal, 41(1), 93-99. http://doi.org/10.1007/s10615012-0405-2

Scheele, D., Schwering, C., Elison, J.T., Spunt, R., Maier, W. \& Hurlemann, R. (2015). A human tendency to anthropomorphize is enhanced by oxytocin. European Neuropsychopharmacol- 
ogy, 25(10), 1817-1823. http://doi.org/10.10 16/j.euroneuro.2015.05.009

Serpell, J.A. (2003). Anthropomorphism and anthropomorphic selection - Beyond the "cute response". Society and Animals, 11(1), 83-100. $\mathrm{http} / / /$ doi.org/10.1163/156853002320936926

Serpell, J.A. (2006). Animal-assisted interventions in historical perspective. En A.H. Fine (Eds.), Handbook on animal-assisted therapy: Theoretical foundations and guidelines for practice (pp. 3-20). London: Academic Press.

Serpell, J. A. \& Paul, E. (2011). Pets in the family: An evolutionary perspective. En C.A. Salmon, \& T.K. Shackelford (Eds.), The Oxford handbook of evolutionary family psychology (pp. 298-309). London: Oxford University Press.

Sprengelmeyer, R., Perrett, D.I., Fagan, E.C., Cornwell, R.E., Lobmaier, J.S., Sprengelmeyer, A. et al. (2009). The cutest little baby face a hormonal link to sensitivity to cuteness in infant faces. Psychological Science, 20(2), 149-154. http://doi.org/10.1111/j.1467-9280.2009.0227 2.x

Swain, J.E., Kim, P., Spicer, J., Ho, S.S., Dayton, C.J., Elmadih, A. \& Abel, K.M. (2014). Ap- proaching the biology of human parental attachment: Brain imaging, oxytocin and coordinated assessments of mothers and fathers. Brain Research, 1580, 78-101. http://doi.org/10.1016/j. brainres.2014.03.007

Uvnäs-Moberg, K., Handlin, L. \& Petersson, M. (2011). Promises and pitfalls of hormone research in human-animal interaction. En P. Mc Cardle, S. McCune, J.A. Griffin, \& V. Maholmes (Eds.), How animals affect us: Examining the influence of human-animal interaction on child development and human health (pp. 5381). Washington: APA.

Valadez Azúa, R. (2000). El origen del perro, primera parte (entre el lobo y el perro) [The origin of the dog, first part (between the wolf and the dog)]. Zootecnia, 11(3), 75-84.

Zilcha-Mano, S., Mikulincer, M. \& Shaver, P.R. (2012). Pets as safe havens and secure bases: The moderating role of pet attachment orientations. Journal of Research in Personality, 46(5), 571-580. http://doi.org/10.1016/j.jrp.2012.06. 005

Universidad de Flores (UFLO) Ciudad Autónoma de Buenos Aires República Argentina

Fecha de recepción: 17 de septiembre de 2015 Fecha de aceptación: 10 de agosto de 2016 